\title{
Malabaricone $C$ as Natural Sphingomyelin Synthase Inhibitor against Diet-Induced Obesity and Its Lipid Metabolism in Mice
}

\author{
3 Muhamad Aqmal Othman, ${ }^{\dagger}$ Kohei Yuyama, ${ }^{\ddagger}$ Yuta Murai, ${ }^{\S}$ Yasuyuki Igarashi, ${ }^{\ddagger}$ Daisuke Mikami, \\ 4 Yasodha Sivasothy, "Khalijah Awang, ${ }^{\perp}$ and Kenji Monde*, ${ }^{\circledR}(0)$ \\ $5{ }^{\dagger}$ Graduate School of Life Science, Hokkaido University, Kita 21 Nishi 11, Sapporo 001-0021, Japan \\ 6 Lipid Biofunction Section, Faculty of Advanced Life Science, Hokkaido University, Kita 21 Nishi 11, Sapporo, 001-0021, Japan \\ $7{ }^{\S}$ Faculty of Advanced Life Science, Hokkaido University, Kita 21 Nishi 11, Sapporo 001-0021, Japan \\ 8 "Research Centre for Crystalline Materials, Faculty of Science and Technology, Sunway University, 47500 Bandar Sunway, Selangor \\ 9 Darul Ehsan Malaysia \\ ${ }_{10}^{\perp}$ Department of Chemistry, Faculty of Science, University of Malaya, 50603, Kuala Lumpur, Malaysia
}

11 S Supporting Information

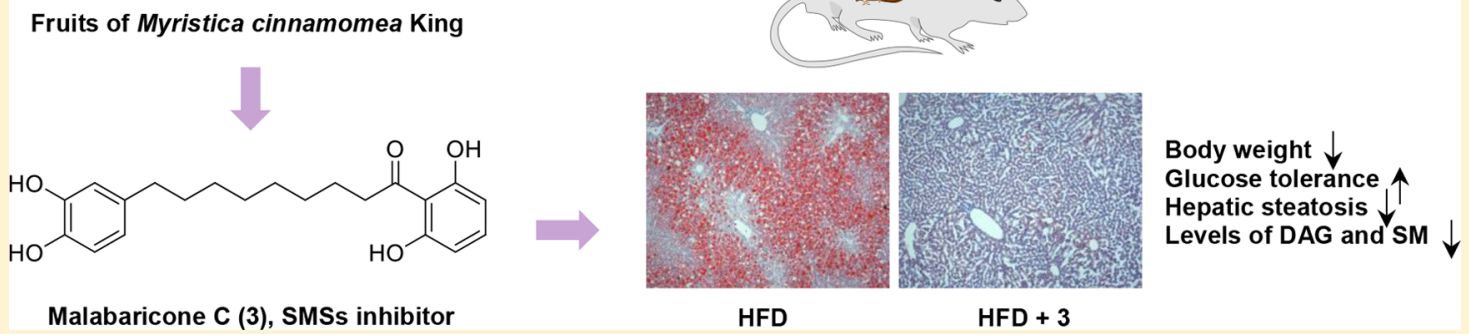

12 ABSTRACT: The interaction between natural occurring inhibitors and targeted membrane proteins could be an alternative 13 medicinal strategy for the treatment of metabolic syndrome, notably, obesity. In this study, we identified malabaricones A-C 14 and E (1-4) isolated from the fruits of Myristica cinnamomea King as natural inhibitors for sphingomyelin synthase (SMS), a 15 membrane protein responsible for sphingolipid biosynthesis. Having the most promising inhibition, oral administration of 16 compound 3 exhibited multiple efficacies in reducing weight gain, improving glucose tolerance, and reducing hepatic steatosis in 17 high fat diet-induced obesity mice models. Liver lipid analysis revealed a crucial link between the SMS activities of compound 3 18 and its lipid metabolism in vitro and in vivo. The nontoxic nature of compound 3 makes it a suitable candidate in search of drugs 19 which can be employed in the treatment and prevention of obesity.

20 KEYWORDS: Membrane protein, sphingomyelin synthase, malabaricone C, myristica cinnamomea, obesity

${ }_{n=12}^{2 !} \mathrm{W}$ orldwide prevalence of obesity has increased substantially over the past 40 years and continues to cause 23 metabolic syndrome, which is associated with dyslipidaemia, 24 insulin resistance, cardiovascular diseases, and type 2 diabetes 25 mellitus (T2DM). ${ }^{1-3}$ These intersecting risks are controlled by 26 a critical and complex metabolic pathway which involves the 27 membrane protein. Having said that, the membrane protein 28 could be the initial key in enhancing the understanding of 29 pharmacology for common metabolic related diseases, notably, 30 obesity. The membrane protein regulates cell communication 31 with its surroundings which is activated by a wide variety of 32 physiological and environmental stimuli including peptides, 33 proteins, small organic molecules, and even ions. ${ }^{4-6}$ About 34 more than $50 \%$ of all known low molecular drugs bind to the 35 membrane protein. ${ }^{7,8}$ Thus, discovering an enzyme inhibitor 36 will be a direct approach in developing low molecular drugs.

37 This study of ours focuses on the sphingomyelin synthase 38 (SMS) membrane protein family which consists of two isozymes, SMS1 and SMS2. ${ }^{9,10}$ Both SMS 1 and 2 catalyze 39 ceramide and phosphatidylcholine (PC) as substrates to 40 produce sphingomyelin (SM) and diacylglycerol (DAG). ${ }^{11,12} 41$ The SMSs modulate SM and other sphingolipids levels, 42 thereby regulating membrane fluidity, ceramide-dependent 43 apoptosis, lipid metabolism, and signal transduction. ${ }^{13-16}$ The 44 increasing levels of SM and DAG produced by the SMSs will 45 lead to obesity and insulin resistance. ${ }^{17,18}$ SMS knockout mice 46 are resistant to Alzheimer's disease, tumorigenesis, diet- 47 induced obesity, and T2DM and are also know to exhibit 48 decreased levels of plasma inflammatory cytokines. ${ }^{19,20,15,21} 49$ Therefore, the inhibition of the SMSs enzymes by natural 50 occurring substrates would be an ideal therapeutic approach 51 for metabolic syndrome.

Received: April 15, 2019

Accepted: June 3, 2019

Published: July 3, 2019 
53 Very recently, the inhibitory activity of gingkolic acid from 54 the leaves of Gingko biloba was reported by our group. ${ }^{22}$ 55 Though, gingkolic acid has been proven to be an effective 56 inhibitor with equal inhibiting potentials $\left(\mathrm{IC}_{50}=1.5 \mu \mathrm{M}\right)$ 57 against both enzymes, studies have revealed that gingkolic acid 58 is toxic, thus making it an unsuitable candidate for the further 59 development of it as a drug. ${ }^{23,24}$ With regard to this, in the 60 present work, we report the isolation of malabaricones $\mathrm{A}-\mathrm{C}$ 61 and $E(1-4)$ as the first naturally occurring SMS inhibitor 62 from edible plants in an effort to display a safe alternative with 63 lesser side effects. ${ }^{25}$ Additionally, we performed a diet-induced 64 obesity test with malabaricone C (3) that showed significant 65 prevention of high fat diet-induced fatty liver.

66 Preliminary screening of the ethyl acetate extract from the 67 fruits of $M$. cinnamomea showed potential inhibitory activity 68 against SMS1 $(13 \mu \mathrm{g} / \mathrm{mL})$ and SMS2 $(10 \mu \mathrm{g} / \mathrm{mL})$, 69 respectively. Therefore, the bioassay-guided fractionation of 70 the extract resulted in the isolation of malabaricones $\mathrm{A}-\mathrm{C}$ and $71 \mathrm{E}(\mathbf{1 - 4})$ as the active compounds (Figure 1). ${ }^{26,27}$

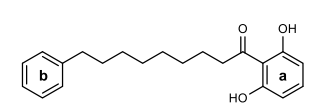

Malabaricone A (1)

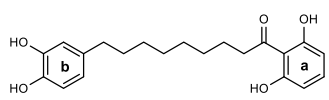

Malabaricone C (3)

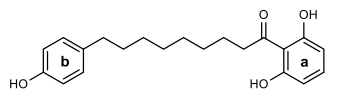

Malabaricone B (2)

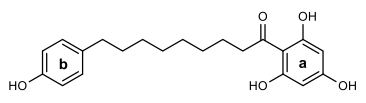

Malabaricone $\mathrm{E}(\mathbf{4})$
Figure 1. Bioassay-guided extraction of the fruits of $M$. cinnamomea afforded four naturally occurring inhibitors for SMS inhibitory activity.

72 Subsequently, compounds $\mathbf{1 - 4}$ were subjected to SMS 73 inhibition assay by lysate-based assay of SMS1- or SMS274 expressed SMS1/2 double knockout mouse fibroblasts. Each of 75 the compounds showed relatively moderate inhibition 76 activities compared with that of previously synthesized 77 inhibitors (Table 1). ${ }^{22}$ A closer look at the structures of

Table 1. Inhibitory Activity of Sphingomyelin Synthase (Lysate Based Assay) $^{a}$

\begin{tabular}{cccc} 
& & \multicolumn{2}{c}{ NBD - Ceramide $\left(\mathrm{IC}_{50}, \mu \mathrm{M}\right)$} \\
\cline { 3 - 4 } No. & Compounds & SMS 1 & SMS 2 \\
$\mathbf{1}$ & Malabaricone A & 4 & 4 \\
$\mathbf{2}$ & Malabaricone B & 3.5 & 2.5 \\
$\mathbf{3}$ & Malabaricone C & 3 & 1.5 \\
$\mathbf{4}$ & Malabaricone E & 6 & 4.5
\end{tabular}

${ }^{a} \mathrm{IC}_{50}$ values are the means of three separate determinations on SMS1 or SMS2 expressed SMS1/2 double knockout mouse fibroblast cell lysate and were determined by more than four concentrations of each inhibitor.

78 compounds 1-4 provided further insight as to how the 79 activities of these compounds might have been influenced by 80 the chemical groups in their respective structures (Figure 1). 81 The SMS inhibiting potentials of compounds $1-3$ could have 82 enhanced with the increase in the number of hydroxyl groups 83 in their ring $b$. The lower SMS inhibiting potentials of 84 compound $\mathbf{4}$ upon comparison to compound $\mathbf{2}$ may have 85 resulted from the additional hydroxyl group in its ring a.
To determine the mode of action for major compounds 1- 86 3, cell lysate assay of the SMS inhibitory activity was carried 87 out by using different substrate concentrations. The $\mathrm{IC}_{50}$ values 88 of 2 to $3 \mu \mathrm{M}$ for SMS1 and 1 to $3 \mu \mathrm{M}$ for SMS2 were obtained 89 in the presence of 5 and $10 \mu \mathrm{M}$ of NDB-Ceramide. As a result, 90 changes in substrate concentration did not significantly affect 91 the $\mathrm{IC}_{50}$ values of compounds $\mathbf{1 - 3}$, thus suggesting that 92 compounds 1-3 were noncompetitive inhibitors of both SMS 93 1 and 2 (Table S1). Cell counting kit- 8 assay was used to 94 evaluate the cytotoxic activity of compound 3 against wild-type 95 mouse embryonic fibroblasts cells, MEF. 56-97\% of the cells 96 were viable after $3 \mathrm{~h}$ of treatment with compound 3 at 97 concentration levels of $1-0.01 \mathrm{mM}$ (Figure S1). Acute toxicity 98 studies of compound 3 at the concentration of $500 \mathrm{mg} / \mathrm{kg} 99$ were previously conducted on mice liver and kidneys. The 100 absent of inflammation, necrosis, and hemorrhaging in the 101 respective organs further supported our findings. ${ }^{28} \quad 102$

Furthermore, in the current investigation, the SMS 103 inhibition assay of compound 3 was carried out with live 104 cells (cell-based assay) and the $\mathrm{IC}_{50}$ values were $13 \mu \mathrm{M}$ and 11105 $\mu \mathrm{M}$ for SMS1 and SMS2 enzymes, respectively (Table S2). 106 These results suggested that compound 3 could be a suitable 107 candidate for further in vitro and in vivo studies based on its 108 previously reported world drug index, Lipinski's rules, 109 nonmutagenicity, and noncarcinogenicity. ${ }^{29}$

It has been reported that a high fat diet (HFD) activates the 111 nuclear receptor PPAR- $\gamma$, which is responsible for the hyper- 112 expression of CD36/FAT. ${ }^{15}$ The SMS2 enzyme facilitates 113 CD36/FAT to take up the PPAR- $\gamma$ ligands, which leads to the 114 accumulation of triglycerides and lipid droplets, thus resulting 115 in fatty liver formation. Since compound 3 exhibited SMS1 and 116 SMS2 inhibitory activities, an oleic acid uptake analysis assay 117 with hepatocytoma HepG2 cells was further conducted to 118 examine the levels of intracellular triglycerides and free fatty 119 acids. Compound 3 decreased the levels of intracellular 120 triglycerides in a dose-dependent manner while it exhibited 121 no significant changes in the free fatty acids levels as compared 122 to the control (Figures $2 \mathrm{~A}-\mathrm{B}$ ). With the promising in vitro $123 \mathrm{f} 2$ effects on oleic acid uptake, we performed a Nile red staining 124 assay to examine the effect of compound 3 on lipid droplet 125 formation in the HepG2 cells. Remarkably, compound 3 for 126 the first time was found to significantly decrease lipid 127 accumulation in a dose-dependent manner (Figures 2C-D). 128 These data indicated that compound 3 was able to prevent 129 cellular uptake by CD36/FAT in a dose dependent manner, 130 which is in good agreement with the results of the previous in 131 vivo effects of SMS2 knockout mice. ${ }^{15}$

With regard to the in vitro results, the selected natural 133 occurring inhibitor was further investigated using (C57BL/6J) 134 mice which were fed with high-fat diet (HFD), normal chow 135 diet (ND), and HFD supplemented with $0.1 \%$ of compound 3. 136 The HFD + 3 mice were healthy and behaved normally over 2137 months with the exception of a noticeably leaner phenotype 138 (Figure 3A). Despite not having any statistically difference in $139 \mathrm{f} 3$ the daily food intake between the HFD control and the HFD +140 3 group (Figure S2), the body weight of the HFD + $\mathbf{3}$ group 141 was significantly lower than those of the controls starting from 142 20 days of treatment with an inclusive weight loss of $42.7 \% 143$ (Figures 3B-C). In addition, an oral glucose tolerance test was 144 performed after 8 weeks of the daily oral administration of the 145 vehicle controls and HFD +3 . Treatment of mice with 146 compound 3 also displayed a more significant improvement in 147 


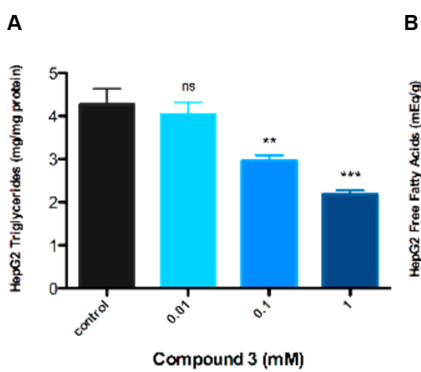

B

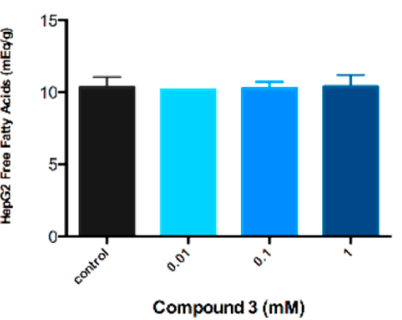

C
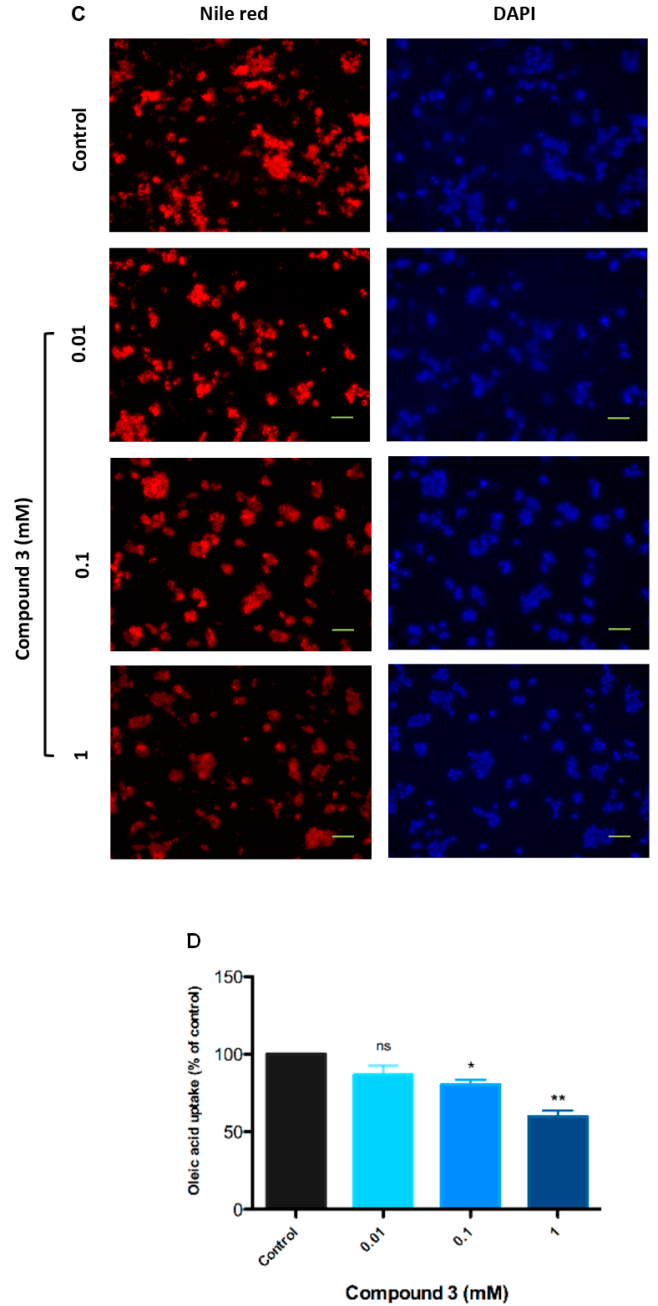

Figure 2. In vitro results of HepG2 cell analysis. Intracellular levels of (A) triglycerides and (B) free fatty acid when treated with different concentrations of compound 3 with oleic acid uptake. (C) Representative images of Nile Red staining and DAPI staining. (D) Oleic acid uptake analysis. Lipid droplets were stained with Nile Red and the numbers of lipid droplets were counted using fluorescent microscopy. Scale bar, $100 \mu \mathrm{m}$. Data are presented as the mean \pm standard error of the mean (SEM). Statistical analysis was done by using $t$ test: $(*) P<0.05$, (**) $P<0.01$, (***) $P<0.001$, (****) $P<$ $0.0005, \mathrm{~ns}=$ no significant difference versus the control.

148 the glucose tolerance than that of the vehicle-treated mice 149 (Figure 3D).

150 The liver plays a key role in lipid metabolism. ${ }^{30}$ Liver weight 151 reduction was observed for the $\mathrm{HFD}+3$ as compared to the

152 HFD group (Figure 4A), but the liver of the HFD +3 group 153 was noticeably redder, possibly implying a decreased fat
A

B
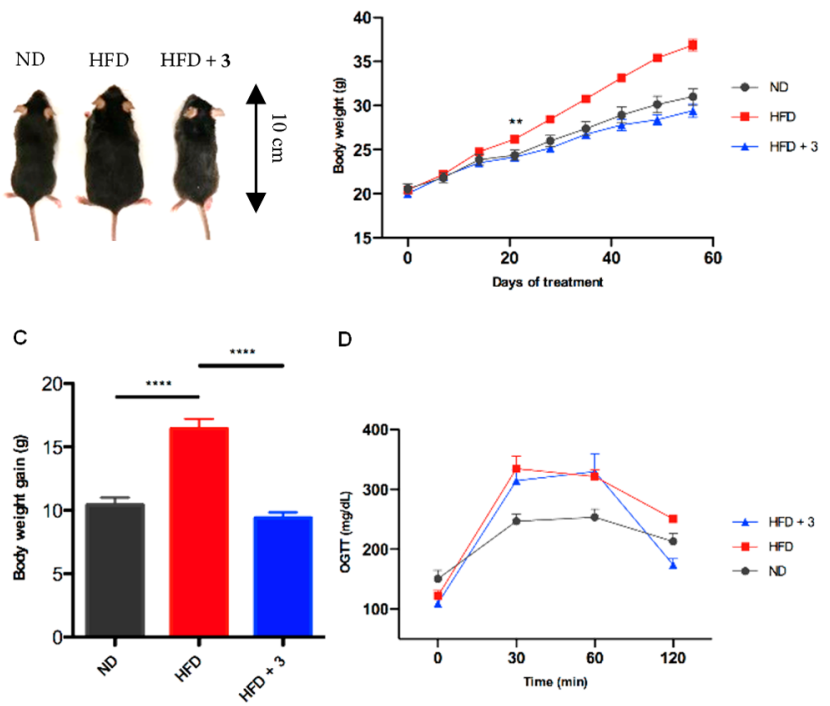

D

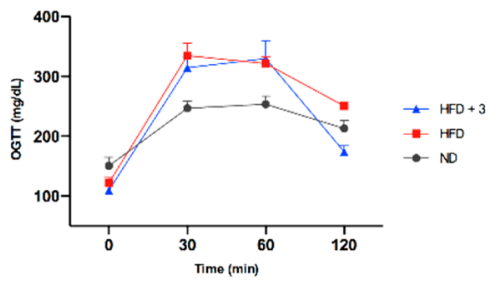

Figure 3. In vivo results of compound 3 on body weight gain and blood glucose levels. (A) Representatives images of the whole mice body. (B-C) Body weight gain. (D) Oral glucose tolerance test. Control: ND, normal chow diet and HFD, High fat diet. Test group: HFD +3 , High fat diet with $0.1 \%$ of Malabaricone C. Data are presented as mean \pm standard error of the mean (SEM); $N=7-8$ mice per group. $t$ test: $(*) P<0.05$, (**) $P<0.01$, (***) $P<0.001$, $(* * * *) P<0.0005$, ns $=$ no significant difference versus the control.

content in the organ (Figure 4B). Previous study has shown 154 that up-regulation of the hepatic lipid metabolism may 155 contribute to the suppression of the liver fat and visceral fat 156 accumulation. ${ }^{31}$ Examination of the histological analysis of the 157 oil red O-stained sectioned of the liver showed the presence of 158 numerous steatosis in the HFD control group as indicated by 159 microscopy observation (Figure 4C). The HFD +3 group on 160 the other hand exhibited resistance in the development of liver 161 steatosis and improved lipid metabolism. Steatosis controls the 162 development of obesity along with metabolic syndrome related 163 disorder. $^{32}$ Consistent with the histochemical results, we found 164 that HFD + 3 effectively reduced the hepatic TG levels (Figure 165 4D). In addition, feeding the mice with HFD + 3 significantly 166 reduced the levels of triglycerides (TG) and free fatty acids 167 (FFAs) in the blood plasma (Figures 4E-F). In comparison 168 with previous plasma free fatty acids in the SMS2 knockout 169 mice in vivo, there is a possibility that the uptake of fatty acids 170 into the liver tissues may not fully be prevented, which further 171 explains the decrease of plasma free fatty acids upon feeding 172 with HFD +3 . Finally, we assessed the synthesis of DAG and 173 SM via liver tissue lysate assays to further confirm the in vivo 174 SMS inhibitory activities by compound 3. Indeed, we have 175 proved that, for the first time, compound 3 as a natural SMS 176 inhibitor, has significantly reduced the synthesis of the DAG 177 and $\mathrm{SM}$ in the liver (Figures $4 \mathrm{G}-\mathrm{H}$ ). Herein, we underlined 178 the in vitro and in vivo efficacies of compound 3 in its 179 inhibition of the SMS2 enzyme and its putative mechanism 180 involving the prevention of obesity. Interestingly, we 181 demonstrated that compound $\mathbf{3}$ results in body weight 182 reduction, improves glucose tolerance, and lowers hepatic 183 steatosis in vivo. Further studies on gene expression related to 184 lipogenesis and gluconeogenesis are required to better 185 understand the exact metabolism which is involved. 
A

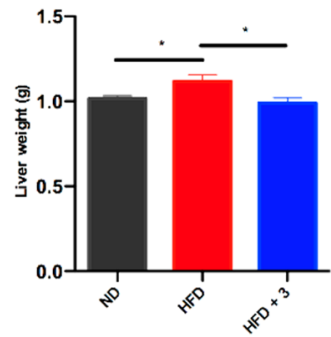

B
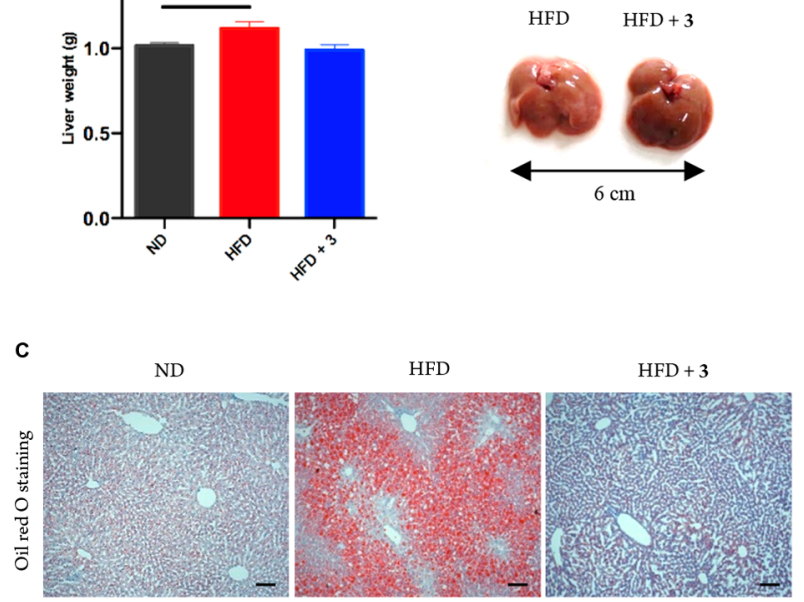

$D$

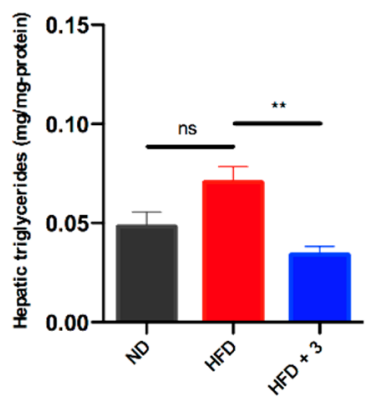

$E$
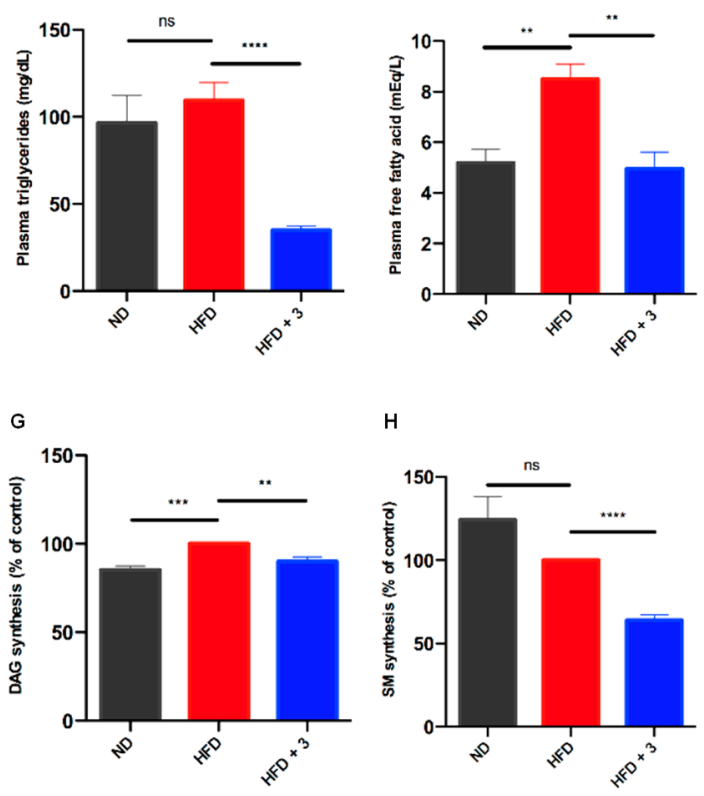

Figure 4. In vivo results of mice liver, lipid metabolism, and SMS inhibitory activity. (A) Liver weight of the mice. (B) Representatives images of liver gross appearance. (C) Representatives images of Oil Red $\mathrm{O}$ staining ( $N=3$ mice per group). (D) Hepatic triglycerides. (E) Plasma triglycerides. (F) Plasma free fatty acids. (G) Conversion of NBD-Phosphocholine. (H) Conversion of NBD-Ceramide. Measurements were taken from distinct samples. Scale bar, $100 \mu \mathrm{m}$. Control: ND, normal chow diet, and HFD, high fat diet; Test group:
Figure 4. continued

HFD +3 , high fat diet with $0.1 \%$ of compound 3 . Data are presented as mean \pm standard error of the mean (SEM); $N=7-8$ mice per group. $t$ test: $(*) P<0.05,(* *) P<0.01,(* * *) P<0.001$, (****) $P$ $<0.0005, \mathrm{~ns}=$ no significant difference versus the control.

In summary, malabaricone C (3), an acylphenol isolated 187 from the fruits of $M$. cinnamomea, has been identified as a lead 188 natural sphingomyelin synthase inhibitor. Having the same 189 mechanisms of action as the previously reported SMS 190 knockout studies, malabaricone C was highly efficacious in 191 preventing oleic acid uptake across the membrane, which in 192 turn reduced lipid droplet formation in vitro. ${ }^{15}$ Malabaricone C 193 was also found to be able to reduce body weight gain, improve 194 glucose tolerance, and decrease lipid accumulation in the liver 195 in vivo, thus making this the first report involving a plant 196 derived SMS inhibitor against high fat diet-induced obesity. Its 197 nontoxic nature makes malabaricone $\mathrm{C}$ a suitable candidate for 198 its further development as a new drug or medicinal supplement 199 to treat and prevent obesity.

\section{ASSOCIATED CONTENT}

200

\section{S Supporting Information}

201

The Supporting Information is available free of charge on the 203 ACS Publications website at DOI: 10.1021/acsmedchem- 204 lett.9b00171.

All experimental procedures, tables on mode of 206 inhibition and cell-based assay inhibition, figures on 207 cytotoxicity of compound 3, and in vivo result for daily 208 food intake (PDF)

\section{9}

\section{AUTHOR INFORMATION}

\section{Corresponding Author}

210

*E-mail: kmonde@sci.hokudai.ac.jp.

ORCID

Author Contributions

214

M.A.O., K.Y., Y.M., and D.M. contributed to the design, 216 execution, and analysis of the in vitro and in vivo experiments. 217 Y.S. and K.A. contributed to plant samples. M.A.O., K.Y., Y.M., 218 Y.S., and K.M. wrote the manuscript. Y.I and K.M. designed 219 and supervised the study. All of the authors have given 220 approval to the final version of the manuscript. 221

\section{Funding}

222

This work was supported by a grant-in-aid for scientific 223 research KAKENHI (grant 23651211 and 17K19188) from 224 the MEXT of Japan and MEXT Doctoral program for Data 225 Related Innovation Expert Hokkaido University (D-DRIVE- 226 $\mathrm{HU})$ program. The authors thank the International Graduate 227 Program (IGP), Hokkaido University for the scholarship given. 228

Notes

The authors declare no competing financial interest.

\section{ACKNOWLEDGMENTS}

We acknowledge a collaborative effort of the International 232 French Malaysia Natural Products Laboratory between CNRS- 233 ICSN and the University of Malaya for providing plant 234 materials (KL5043). We would like to thank Yoshiko Suga and 235 Prof. Anetai Masaki who performed the screening experiments. 236 
237 This work was inspired by JSPS Asian CORE Program "Asian 238 Chemical Biology Initiative” and JSPS A3 Foresight Program.

\section{$239 \square$ ABBREVIATIONS}

240 SMS, sphingomyelin synthase; PC, phosphatidylcholine; SM, 241 sphingomyelin; DAG, diacylglycerol; ND, normal chow diet; 242 HFD, high fat diet; PPAR- $\gamma$, peroxisome proliferator-activated 243 receptor gamma; CD36, cluster of differentiation; FAT, fatty 244 acid translocase; TG, triglycerides; FFA, free fatty acid; NBD, $245 \mathrm{~N}$-[6-[(7-nitro-2-1,3-benzoxadiazol-4-yl)amino]hexanoyl]; 246 T2DM, type 2 diabetes mellitus; OGTT, oral glucose tolerance 247 test; DAPI, 4',6-diamidino-2-phenylindole.

\section{$248 \square$ REFERENCES}

249 (1) Jaacks, L. M.; Vandevijvere, S.; Pan, A.; McGowan, C. J.; 250 Wallace, C.; Imamura, F.; Mozaffarian, D.; Swinburn, B.; Ezzati, M. 251 The Obesity Transition: Stages of the Global Epidemic. Lancet 252 Diabetes Endocrinol. 2019, 7 (3), 231-240.

253 (2) Wang, K.; Bao, L.; Zhou, N.; Zhang, J.; Liao, M.; Zheng, Z.; 254 Wang, Y.; Liu, C.; Wang, J.; Wang, L.; et al. Structural Modification of 255 Natural Product Ganomycin I Leading to Discovery of a $\alpha$ 256 Glucosidase and HMG-CoA Reductase Dual Inhibitor Improving 257 Obesity and Metabolic Dysfunction in Vivo. J. Med. Chem. 2018, 61 258 (8), 3609-3625.

259 (3) De Haas, E. C.; Oosting, S. F.; Lefrandt, J. D.; Wolffenbuttel, B. 260 H. R.; Sleijfer, D. T.; Gietema, J. A. The Metabolic Syndrome in 261 Cancer Survivors. Lancet Oncol. 2010, 11 (12), 193-203.

262 (4) Goto-Inoue, N.; Yamada, K.; Inagaki, A.; Furuichi, Y.; Ogino, S.; 263 Manabe, Y.; Setou, M.; Fujii, N. L. Lipidomics Analysis Revealed the 264 Phospholipid Compositional Changes in Muscle by Chronic Exercise 265 and High-Fat Diet. Sci. Rep. 2013, 3 (1), 3267.

266 (5) Lee, Y.; Basith, S.; Choi, S. Recent Advances in Structure-Based 267 Drug Design Targeting Class A G Protein-Coupled Receptors 268 Utilizing Crystal Structures and Computational Simulations. J. Med. 269 Chem. 2018, 61 (1), 1-46.

270 (6) Overington, J. P.; Al-Lazikani, B.; Hopkins, A. L. How Many 271 Drug Targets Are There? Nat. Rev. Drug Discovery 2006, 5 (12), $993-$ 272996.

273 (7) Hopkins, A. L.; Groom, C. R. The Druggable Genome. Nat. Rev. 274 Drug Discovery 2002, 1 (9), 727-730.

275 (8) Berridge, G.; Chalk, R.; D’Avanzo, N.; Dong, L.; Doyle, D.; Kim, 276 J.-I.; Xia, X.; Burgess-Brown, N.; deRiso, A.; Carpenter, E. P.; et al. 277 High-Performance Liquid Chromatography Separation and Intact 278 Mass Analysis of Detergent-Solubilized Integral Membrane Proteins. 279 Anal. Biochem. 2011, 410 (2), 272-280.

280 (9) Huitema, K.; van den Dikkenberg, J.; Brouwers, J. F. H. M.; 281 Holthuis, J. C. M. Identification of a Family of Animal Sphingomyelin 282 Synthases. EMBO J. 2004, 23 (1), 33-44.

283 (10) Yamaoka, S.; Miyaji, M.; Kitano, T.; Umehara, H.; Okazaki, T. 284 Expression Cloning of a Human CDNA Restoring Sphingomyelin 285 Synthesis and Cell Growth in Sphingomyelin Synthase-Defective 286 Lymphoid Cells. J. Biol. Chem. 2004, 279 (18), 18688-18693.

287 (11) Ullman, M. D.; Radin, N. S. The Enzymatic Formation of 288 Sphingomyelin from Ceramide and Lecithin in Mouse Liver. J. Biol. 289 Chem. 1974, 249 (5), 1506-1512.

290 (12) Merrill, A. H.; Jones, D. D. An Update of the Enzymology and 291 Regulation of Sphingomyelin Metabolism. Biochim. Biophys. Acta, 292 Lipids Lipid Metab. 1990, 1044 (1), 1-12.

293 (13) Bienias, K.; Fiedorowicz, A.; Sadowska, A.; Prokopiuk, S.; Car, 294 H. Regulation of Sphingomyelin Metabolism. Pharmacol. Rep. 2016, 29568 (3), 570-581.

296 (14) Hannun, Y. A. Functions of Ceramide in Coordinating Cellular 297 Responses to Stress. Science (Washington, DC, U. S.) 1996, 274 298 (5294), 1855-1859.

299 (15) Mitsutake, S.; Zama, K.; Yokota, H.; Yoshida, T.; Tanaka, M.; 300 Mitsui, M.; Ikawa, M.; Okabe, M.; Tanaka, Y.; Yamashita, T.; et al. 301 Dynamic Modification of Sphingomyelin in Lipid Microdomains
Controls Development of Obesity, Fatty Liver, and Type 2 Diabetes. 302 J. Biol. Chem. 2011, 286 (32), 28544-28555.

(16) Yuyama, K.; Sun, H.; Mitsutake, S.; Igarashi, Y. Sphingolipid- 304 Modulated Exosome Secretion Promotes Clearance of Amyloid- $\beta$ by 305 Microglia. J. Biol. Chem. 2012, 287 (14), 10977-10989.

306

(17) Hanamatsu, H.; Ohnishi, S.; Sakai, S.; Yuyama, K.; Mitsutake, 307 S.; Takeda, H.; Hashino, S.; Igarashi, Y. Altered Levels of Serum 308 Sphingomyelin and Ceramide Containing Distinct Acyl Chains in 309 Young Obese Adults. Nutr. Diabetes 2014, 4 (10), No. e141. 310

(18) Kim, Y.-J.; Greimel, P.; Hirabayashi, Y. GPRC5B-Mediated 311 Sphingomyelin Synthase 2 Phosphorylation Plays a Critical Role in 312 Insulin Resistance. iScience 2018, 8, 250-266.

313

(19) Yuyama, K.; Mitsutake, S.; Igarashi, Y. Pathological Roles of 314 Ceramide and Its Metabolites in Metabolic Syndrome and 315 Alzheimer's Disease. Biochim. Biophys. Acta, Mol. Cell Biol. Lipids 316 2014, 1841 (5), 793-798.

(20) Ohnishi, T.; Hashizume, C.; Taniguchi, M.; Furumoto, H.; 318 Han, J.; Gao, R.; Kinami, S.; Kosaka, T.; Okazaki, T. Sphingomyelin 319 Synthase 2 Deficiency Inhibits the Induction of Murine Colitis- 320 Associated Colon Cancer. FASEB J. 2017, 31 (9), 3816-3830. 321

(21) Fan, Y.; Shi, F.; Liu, J.; Dong, J.; Bui, H. H.; Peake, D. A.; Kuo, 322 M.-S.; Cao, G.; Jiang, X.-C. Selective Reduction in the Sphingomyelin 323 Content of Atherogenic Lipoproteins Inhibits Their Retention in 324 Murine Aortas and the Subsequent Development of Atherosclerosis. 325 Arterioscler., Thromb., Vasc. Biol. 2010, 30 (11), 2114-2120. 326

(22) Swamy, M. M. M.; Murai, Y.; Ohno, Y.; Jojima, K.; Kihara, A.; 327 Mitsutake, S.; Igarashi, Y.; Yu, J.; Yao, M.; Suga, Y.; et al. Structure- 328 Inspired Design of a Sphingolipid Mimic Sphingosine-1-Phosphate 329 Receptor Agonist from a Naturally Occurring Sphingomyelin 330 Synthase Inhibitor. Chem. Commun. 2018, 54 (90), 12758-12761. 331

(23) Liu, Z. H.; Zeng, S. Cytotoxicity of Ginkgolic Acid in HepG2 332 Cells and Primary Rat Hepatocytes. Toxicol. Lett. 2009, 187 (3), 333 $131-136$.

(24) Jiang, L.; Si, Z.-H.; Li, M.-H.; Zhao, H.; Fu, Y.-H.; Xing, Y.-X.; 335 Hong, W.; Ruan, L.-Y.; Li, P.-M.; Wang, J.-S. 1H NMR-Based 336 Metabolomics Study of Liver Damage Induced by Ginkgolic Acid 337 (15:1) in Mice. J. Pharm. Biomed. Anal. 2017, 136, 44-54. 338

(25) Seidemann, J. World Spice Plants: Economic Usage, Botany, 339 Taxonomy; Springer-Verlag, 2005; pp 215-248. 340

(26) Purushothaman, K. K.; Sarada, A.; Connolly, J. D.; 341 Malabaricones, A-D. Novel Diarylnonanoids from Myristica 342 Malabarica Lam (Myristicaceae). J. Chem. Soc., Perkin Trans. 1343 1977, 0 (5), 587-588.

(27) Abdul Wahab, S. M.; Sivasothy, Y.; Liew, S. Y.; Litaudon, M.; 345 Mohamad, J.; Awang, K. Natural Cholinesterase Inhibitors from 346 Myristica Cinnamomea King. Bioorg. Med. Chem. Lett. 2016, 26 (15), 347 3785-3792.

(28) Banerjee, D.; Maity, B.; Bandivdeker, A. H.; Bandyopadhyay, S. 349 K.; Chattopadhyay, S. Angiogenic and Cell Proliferating Action of the 350 Natural Diarylnonanoids, Malabaricone B and Malabaricone C during 351 Healing of Indomethacin-Induced Gastric Ulceration. Pharm. Res. 352 2008, 25 (7), 1601-1609.

(29) Riju, A.; Sithara, K.; Nair, S. S.; Shamina, A.; Eapen, S. J. In 354 Silico Screening Major Spice Phytochemicals for Their Novel 355 Biological Activity and Pharmacological Fitness. J. Bioequiv. Availab. 356 2009, 01 (02), 1-11.

(30) Zhang, L.; Wang, D.; Wen, M.; Du, L.; Xue, C.; Wang, J.; Xu, 358 J.; Wang, Y. Rapid Modulation of Lipid Metabolism in C57BL/6J 359 Mice Induced by Eicosapentaenoic Acid-Enriched Phospholipid from 360 Cucumaria Frondosa. J. Funct. Foods 2017, 28, 28-35.

361

(31) Murase, T.; Nagasawa, A.; Suzuki, J.; Hase, T.; Tokimitsu, I. 362 Beneficial Effects of Tea Catechins on Diet-Induced Obesity: 363 Stimulation of Lipid Catabolism in the Liver. Int. J. Obes. 2002, 26364 (11), 1459-1464.

(32) Diehl, A. M. Fatty Liver, Hypertension, and the Metabolic 366 Syndrome. Gut 2004, 53 (7), 923-924. 